\title{
EDITORIAL
}

\section{Molecular Psychiatry: Twenty years of progress from bench to clinic}

\section{Molecular Psychiatry (2015) 20, 657; doi:10.1038/mp.2015.60}

The field of psychiatry has evolved immensely in the past 20 years, our journal's life span. This issue-part of our commemorative twentieth-anniversary volume-attests to such progress, with article titles that speak for themselves, revealing the amazing science that underlies contemporary psychiatry, and its immediate relevance to clinical practice:

- 'Lithium in the treatment of bipolar disorder: pharmacology and pharmacogenetics'

- 'Kraepelin revisited: schizophrenia from degeneration to failed regeneration'

- 'Noncoding RNAs and neurobehavioral mechanisms in psychiatric disease'

- 'Adjunctive raloxifene treatment improves attention and memory in men and women with schizophrenia'

- 'Estradiol for treatment-resistant schizophrenia: a large-scale randomized controlled trial in women of child-bearing age'

- 'Characterization of bipolar disorder patient-specific induced pluripotent stem cells from a family reveals neurodevelopmental and mRNA expression abnormalities'

- 'Mental disorders and risk of suicide attempt: a national prospective study'

- 'Prenatal antidepressant exposure is associated with risk for attention-deficit hyperactivity disorder but not autism spectrum disorder in a large health system'

- 'The association between lower educational attainment and depression owing to shared genetic effects? Results in $\sim 25000$ subjects'

- 'Neurotrophic factor-a1 prevents stress-induced depression through enhancement of neurogenesis and is activated by rosiglitazone'

- 'Mind the gap: glucocorticoids modulate hippocampal glutamate tone underlying individual differences in stress susceptibility'

- 'Gray-matter volume, midbrain dopamine D2/D3 receptors and drug craving in methamphetamine users'

- 'Telling true from false: cannabis users show increased susceptibility to false memories'

- 'Cigarette smoking and thinning of the brain's cortex'

- 'Genetic background of extreme violent behavior'

The titles above would fit very nicely in the New York Times's new app for the Apple Watch, in which one-sentence summaries of news articles appear on the watch's screen. The summaries must be compelling and self-contained but also whet the viewer's appetite for the source material. Appealing sentences are difficult to generate. There is a legend that, while lunching with friends at a restaurant, Nobel laureate Ernest Hemingway bet each person at the table $\$ 10$ that he could tell an entire story in six words. He won, with 'For sale. Baby shoes. Never worn.' The Gotham Writers' Workshop recently ran a competition for short stories told in 10 words or fewer. Our entry-'As I strangled Dad, he knew why he was dying' - was not one of the finalists. However, the ' $I$ ' in that story could easily have been a subject in the study 'Genetic background of extreme violent behavior' (786-792).

There is, in both the criminal system and society, a perspective that all behavior, particularly violent criminal behavior that injures third parties, is a choice, a direct outcome of the concept of 'free will' for which culprits need to pay with long periods of incarceration or with their lives. Putting millennia of dogma aside, what if violent behavior is not a choice stemming from free will but, instead, the outcome of genetics? This paradigm shift is addressed in this issue by Tiihonen et al.

To expand this paradigm, what if addiction is not a choice? Morales et al. (658 and 764-771) used positron emission tomography to examine brain dopamine receptors during drug craving in methamphetamine users. Two other intriguing papers address substance abuse: Riba et al. (772-777) demonstrate that smoking marijuana facilitates the emergence of false memories, and Karama et al. (778-785) found thinning of the brain cortex as a result of cigarette smoking. A provocative possibility is that as substances are used, they damage the brain, making 'free will' and 'behavior as the outcome of choice' even more remote. Taking this line of reasoning further, one could argue that genes and prenatal environment (see Clements and colleagues' article on an association of prenatal antidepressant exposure with attentiondeficit hyperactivity disorder; 727-734) compel behaviors. Some of those behaviors, e.g., addiction, may damage the brain and become self-perpetuating. What about choice?

Furthermore, what choice do those with schizophrenia, bipolar disorder, depression, and suicidality (as discussed by Hoertel et al.; 718-726) really have? When they are clinically evident, those highly disabling disorders have a devastating impact. More precise use of existing treatments, including lithium (see the article by Alda; 661-670), and new therapeutic strategies (presented by Weickert et al. (685-694), Kulkarni et al. (695-702), and Cheng et al. (744-754)) could drastically improve outcomes. Finally, new approaches, such as the use of patient-specific induced pluripotent stem cells, can reveal patient- and family-specific abnormalities (discussed by Madison et al. (703-717) in the issue's cover article).

We are delighted that the papers by Weickert et al. (685-694) and Hoertel et al. (718-726) were highlighted at a special session at the Annual Meeting of the American Psychiatry Association in Toronto on May 18, 2015, jointly with two other papers from the American Psychiatric Association.

The extent of progress in psychiatry today is just staggering. We are happy and proud that some of the best work in our field appears in the pages of Molecular Psychiatry.

\section{CONFLICT OF INTEREST}

The authors declare no conflict of interest.

J Licinio and M-L Wong South Australian Health and Medical Research Institute and Department of Psychiatry, School of Medicine, Flinders University, Adelaide, SA, Australia E-mail: julio.licinio@sahmri.com 